\title{
Cutaneous Stem Cells and Wound Healing
}

\author{
CECILIA ROH AND STEPHEN LYLE \\ Department of Pathology [C.R.], Beth Israel Deaconess Medical Center, Boston, MA, 02215; Departments of Cancer Biology and \\ Pathology [S.L.], University of Massachusetts Medical School, Worcester, MA 010605
}

\begin{abstract}
In the last two decades, there has been a tremendous increase in the understanding of stem cell biology, including the field of cutaneous stem cells. Extensive stem cell research and potential clinical applications have provided new perspectives in the use of stem cells in the treatment of human skin disorders such as severe burns and wounds, as well as skin cancer and alopecia. Adult, tissue-specific stem cells are required for tissue homeostasis as well as for the ability to respond to insults such as during wound healing. Fetal wounds can heal rapidly without scars, while in adults wound healing decreases with aging, and this likely represents changes in the functional status of stem cells. In this review, we summarize the main characteristics of cutaneous stem cells in general and present the most recent knowledge in our understanding of these stem cells. We also address the difference in tissue regeneration between fetal and adult tissues in the aspect of stem cell biology.
\end{abstract}

(Pediatr Res 59: 100R-103R, 2006)

$\mathrm{T}$ he essential functions of the skin involve the homeostatic mechanisms of thermoregulation, maintenance of fluid balance, and protection against a variety of environmental insults (1). As a continuously renewing tissue consisting of different specialized epithelial (epidermis, hair follicle and glands) and stromal cell compartments, skin maintenance under steady-state conditions is dependent on tissue-specific (adult-type) stem cells. In the skin there appears to be a hierarchy of stem cells, so that under normal conditions, each compartment is likely maintained by lineage-restricted stem cells or precursors, while multi-potent stem cells can serve as a reservoir of cells when needed. After injury, tissue regeneration requires the activation of stem cells, and it appears that multi-potent stem cells are mobilized $(2,3)$. Interestingly, the result of injury differs dramatically between fetal and adult skin, i.e. in scarring after tissue damage (4). What follows is a review of the characteristics, development, and maintenance of cutaneous stem cells in general, as well as their role in wound healing and tissue regeneration.

\section{CUTANEOUS ANATOMY}

Knowledge of skin anatomy and physiology with respect to stem cells is important for understanding skin diseases and tissue response to injury. There have been significant advances in our understanding of the anatomy of skin with regards to the location and organization of stem cells which are summa-

Received November 15, 2005; accepted December 13, 2005.

Correspondence: Stephen Lyle, M.D., Ph.D., University of Massachusetts Medical School, 364 Plantation St., LRB 411, Worcester, MA 01605; e-mail: stephen.lyle@ umassmed.edu.

Supported by NIH HL007893 (C.R.), NIH AR02179 (S.L.) and the Ellison Medical Foundation.

DOI: $10.1203 / 01 . p d r .0000203572 .51876 . b a$ rized in Figure 1. The cutaneous epithelium consists of epidermis, hair follicles, sebaceous glands and sweat glands. The epidermis is a stratified epithelium composed of keratinocytes. Basal cells are mitotically active, but they lose this potential when they detach from the basement membrane and migrate toward the skin surface in the process of terminal differentiation. Such stratification of the epidermis starts during embryonic development and it continues to occur throughout the life of the organism. The sebaceous gland is composed of a thin rim of progenitor basaloid cells and sebocytes which accumulate lipid and undergo holocrine secretion to produce the sebum (5). The sebaceous gland empties into the upper follicle through a duct that is connected to the follicular epithelium. The hair follicle is composed of an outer root sheath that is contiguous with the epidermis, an inner root sheath and the hair shaft. The matrix contains actively dividing, relatively undifferentiated cells that surround the specialized, mesenchymal dermal papilla cells. Unlike the epidermis and sebaceous gland which are continuously renewing, the hair follicle cycles through periods of active growth (anagen), regression (catagen), rest (telogen) and regeneration (anagen onset) (6). Each of these epithelial compartments appears to contain lineage-restricted stem cells or precursors. Multi-potent cutaneous stem cells are located in a specialized region (niche) within the outer root sheath of the hair follicle known as the bulge (7-9). Interestingly, melanocyte stem cells/precursors have also been localized in the bulge region (10). The hair follicle bulge has many features of a stem cell niche such as its location in a well-protected area, which is highly vascularized, and innervated.

\section{IDENTIFICATION OF CUTANEOUS EPITHELIAL STEM CELLS}

Stem cells are defined by their unique characteristics which include: 1) self-renewal, the ability to divide and generate one daughter cell that retains the parent stem cell phenotype and one transit-amplifying (TA) cell which undergoes rapid proliferation and differentiation to replenish the cells that are lost to the environment after terminal differentiation; 2) slow-cycling nature in vivo; 3) high proliferative potential; 4) participation in tissue regeneration and repair by proliferating at times of tissue expansion such as during fetal development and wound healing; 5) maintenance of somatic homeostasis in vivo; 6) multi-potential

Abbreviations: K15, keratin 15; LRCs, label-retaining cells; TA, transitamplifying; TGF $\boldsymbol{\beta}$, transforming growth factor- $\beta$. 


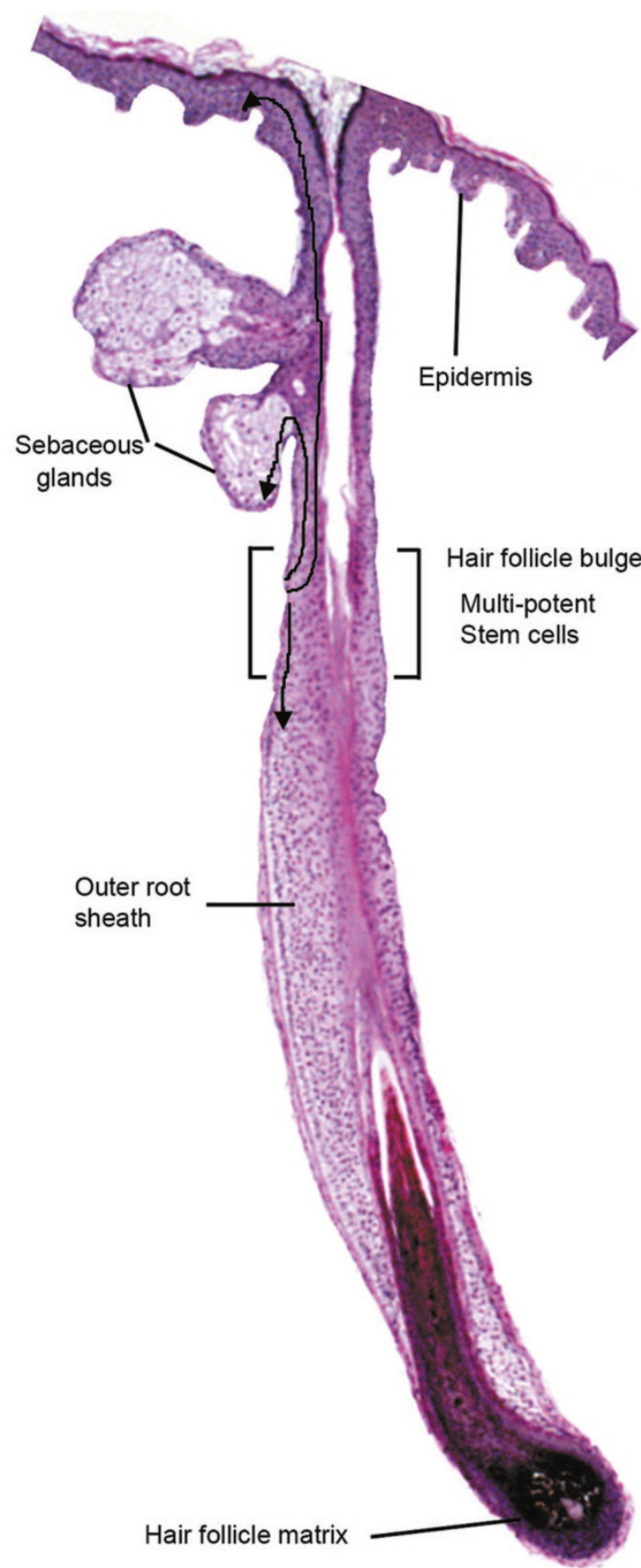

Figure 1. Schematic representation of the cutaneous epithelium and the cell lineages derived from multi-potent stem cells in the hair follicle bulge. Under steady-state conditions, the stem cells are quiescent. At the beginning of the hair cycle when the lower follicle and matrix is regenerated, the stem cells in the bulge proliferate to give rise to new hair follicle keratinocytes. Under wounding conditions, the stem cells produce daughter cells that migrate from the niche to re-populate the basal layer of the epidermis, and the sebaceous gland.

capacity to differentiate to more than one cell lineage. These properties will be described in more detail below.

Two major criteria to define stem cells are slow-cycling nature and high proliferative potential. These properties are somewhat interrelated and can be examined by in vivo and in vitro methodologies. Pulse-chase labeling with a pulse of bromodexoyuridine or tritiated thymidine labels the DNA of actively proliferating cells. During the chase period, the label is diluted in rapidly dividing cells while terminally differentiating cells are lost to the environment. Thus, after the chase period, only slowly-cycling cells which retain the label are detected. Studies in murine skin have demonstrated label-retaining cells in both the epidermis and hair follicle bulge $(7,11,12)$ although the hair follicle bulge cells are the slowest-cycling cells in cutaneous epithelium (13). Using skin transplantation onto SCID mice and pulse-chase labeling, we showed that the same bulge region in human skin also contains label-retaining cells (14).

The proliferative potential of keratinocytes in vitro can be estimated by the size of colonies that they are able to generate (15). Holoclones, which are felt to represent stem cells, have high reproductive capacity and low level of terminal differentiation. Paraclones contain exclusively cells with a short replicative lifespan, while meroclones contain a mixture of cells of different growth potential and represent a transitional stage between holoclones and paraclones. Another indicator of proliferative capability is colony forming efficiency (colonies per number of cells plated) that is thought to correlate to the number of stem cells in a tissue $(16,17)$. These types of analyses apply well for human epidermis where there are cells with three apparent types of proliferative capacity. Terminally differentiated cells have no proliferative capacity, TA cells have a limited capacity (forming abortive small colonies) and epidermal progenitor cells have clonogenic capacity $(16,18)$. The human hair follicle appears more complex. The stem cells in the bulge region are normally quiescent and only proliferate at the onset of anagen when a new lower follicle is regenerated (8). The daughter cells in the lower follicle then must maintain hair growth for the three- to eight-year hair growth cycle on the human scalp. Colony forming ability has been demonstrated for keratinocytes throughout the entire length of the hair follicle including the bulge $(19,20)$, however most of the cells extracted from the lower follicle represent meroclones and cells with the longest in vitro lifespan were found in the region containing the bulge. We have shown that matrix TA cells can form colonies and can be serially-passaged in culture, indicating an extensive proliferative capacity, however stem cells from the bulge possess a greater proliferative capacity and longer in vitro life-span (21). Thus the evidence suggests that the human hair follicle contains 1) stem cells with the greatest proliferative capacity; 2) early TA cells of the lower follicle with extensive but finite proliferative potential; 3) TA cells (similar to epidermal TA cells) with very limited growth potential; and 4) terminally differentiated cells.

Recent advances in methods to isolate cutaneous stem cells have lead to a greater understanding of the multi-potentiality of these cells. Using transplantation of the murine bulge region, Oshima et al. demonstrated that bulge cells could repopulate the epidermis, sebaceous gland and all epithelial layers of the hair follicle (22). Lineage analysis of isolated murine bulge cells in a skin reconstitution system showed that they could regenerate all epithelial cells layers of skin (9). Human adult epithelial stem cells may not be as permissive as rodent cells however, since no successful skin reconstitution assays have been reported. We have shown that stem cells extracted from the human bulge region can be induced to exhibit hair follicle differentiation in vitro (23), as well as form an epidermis and sebaceous cells in vitro (unpublished data), and this supports the multi-potential capacity of human cutaneous stem cells. In addition, cells from the hair follicle are currently used clinically to generate epithe- 
lium used for chronic wound coverage. The above evidence has overwhelmingly demonstrated that the cells located in the hair follicle bulge region represent multi-potent, tissue-specific epithelial stem cells.

\section{STEM CELL MARKERS}

In an effort to understand what genes define the stem cell phenotype and control stem cell properties, as well as to develop improved methods for stem cell isolation, a number of studies have focused on determining stem cell specific markers. $\beta 1$ integrin was the first marker shown to be over-expressed in epidermal stem cells compared to TA cells (16). It is also more highly expressed in multi-potent stem cells in the hair follicle bulge (8). Cell sorting using $\alpha 6$-integrin bright/CD71 (transferrin receptor) dim has proven a good method to purify epidermal stem cells $(24,25)$. Notably, expression of the integrins $\alpha 6$ and $\beta 1$ has been reported in two separate studies investigating the transcriptional profile of several lineages of adult and embryonic stem cells, the putative molecular signature of "stemness" $(26,27)$. In addition, the ability of stem cells to adhere more rapidly to type IV collagen has been used to enrich for cutaneous stem cells $(16,21)$. Keratin 15 (K15) expression was found to be highly specific for stem cells in the human hair bulge (8), and promoter analysis in transgenic mice has demonstrated its utility for isolating a purified population of multi-potent stem cells for a variety of studies $(3,8,28)$. A number of other genes have been found to be up-regulated in cutaneous stem cells including $\Delta$ Np63 (29), $\beta$-catenin (30), melanoma-associated chondroitin sulphate proteoglycan (31), CD 34 (9,32), cyclin-D1 (33) and CD24 (34). The most exciting advances in defining the cutaneous epithelial stem cell transcriptome come from recent studies by the groups of Fuchs (32) and Cotsarelis (9) who have reported the isolation, from the bulge region, of cell populations that are enriched for putative stem cells. The phenotypic and functional characterization of these cells both in vitro and in vivo indicates that they meet many of the hallmark definitions of stem cells.

\section{KERATINOCYTES AND WOUND HEALING}

The normal process of keratinocyte differentiation is to maintain the epidermis, an important barrier between the organism and its environment. This regular self-renewal of keratinocytes can be interrupted when an injury occurs which is followed by the wound healing process. Wound healing is a result of a highly coordinated series of events, initiated by formation of a fibrin clot followed by recruitment of inflammatory cells, formation of granulation tissue with anigiogenesis, fibroblast proliferation, and migration of keratinocytes, contraction of the dermis, and scar remodeling (4). Although both fetal and adult tissues are able to repair a wound in the same quality of healing, the differences lie for its temporal delay and scarring in adult tissue (35). Since this observation was first reported in 1979 (36), there has been an intense research to elucidate the mechanisms underlying scarless fetal wound repair, however many questions remain.

In response to injury, keratinocytes are first to respond as they represent the body's first line of defense from the outside offensive environment. After injury, keratinocytes become activated and secrete various cytokines and growth factors (37). Depending on the types of injuries, keratinocytes first assess the damage and appropriately respond to it. During the process of tissue repair, keratinocytes are required to activate, migrate, and differentiate for a proper wound healing process.

\section{WOUND HEALING IN ADULT AND FETAL TISSUES}

Fetal tissue has the ability to rapidly heal skin wounds without a scar unlike adult tissue. A number of mechanistic differences between fetal and adult wound healing have been identified (4). For instance, fetal tissue is in a sterile environment with amniotic fluid while adult tissue is dry and contaminated. In fetal skin, there is rapid re-epithelialization and the skin is less differentiated. Fetal skin has more hyaluronic acid and nonsulfated glycosaminoglycans. In fetal wounds there is little inflammation compared with adult wounds. In fetal wound healing, transforming growth factor- $\beta 3$ (TGF $\beta$ ) isoform is over-expressed and fibromodulin is the predominant TFG $\beta$ modulator, while adult healing demonstrates TFG $\beta 1$ isoform expression and decorin is the predominant TGF $\beta$ modulator. In fetal stroma there is more fibronectin and tenascin, more fibroblast migration and a higher proportion of collagen type III than in adult scars. Much of the evidence indicates that scarless wound healing appears to be intrinsic to fetal skin and independent of the intrauterine environment. Differences in stem cells between fetal and adult skin in the context of wound healing has not been extensively studied, however recent work suggests that this is an important area for further investigation.

\section{STEM CELLS, AGING AND TISSUE REGENERATION}

Earlier wounding and pulse-chase labeling studies showed that stem cells in the bulge were activated to proliferate and generate cells that migrated up the follicular infundibulum toward the epidermis (2). Recent fate-mapping studies showed that cells derived from stem cells in the bulge migrated into the epidermis and toward the center of an acute cutaneous wound (3). However, epidermal stem cells also responded to the wound and persisted while the bulge-derived cells were only transiently present. It thus appears that both epidermal stem cells as well as the reservoir of multi-potent stem cells in the bulge are used for efficient wound healing. A detailed investigation of epithelial stem cell status between fetal and adult wounding models has not yet been undertaken.

In our analysis of the K15 promoter we showed that it is distinctly active in the hair follicle bulge of mature murine skin (8). Surprisingly, K15 promoter activity showed dramatic changes within the epidermis as the mice aged. Confluent basal epidermal expression of $\mathrm{K} 15$ is seen in neonatal mouse skin by in situ hybridization and in K15-LacZ transgenic mice, but expression gradually decreased with age (28). This pattern also correlates with protein expression. As shown in Fig. 2, young mice express K15 throughout the basal layer of the epidermis as well as in the bulge. As mice age, there is a dramatic decrease in epidermal expression, while the bulge remains strongly positive. It is well known that levels of functional epidermal stem cells decreases with aging in human skin (15). However, it is still unclear if human fetal skin differs in stem cell number, location and function from adult skin, and when the changes occur. Similar to neonatal mouse skin, human fetal skin also shows confluent basal epidermal expression of the K15 stem cell marker (Fig. 2). These results 


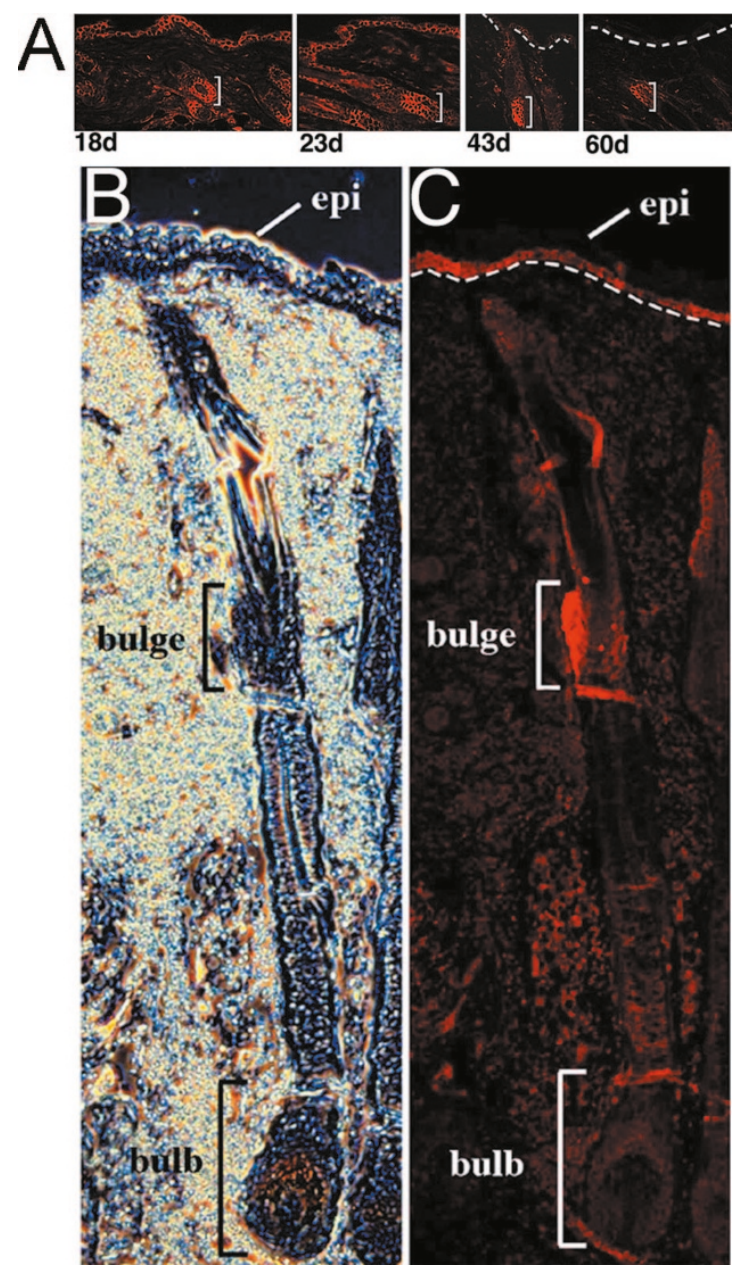

Figure 2. K15 immunofluorescence staining of skin at different ages. A) Mouse skin at indicated ages were stained for keratin 15 (DAKO, clone C8/144B). The staining pattern of K15 in the basal layer correlates with promoter activity. Human fetal skin at week 17 was imaged with phasecontrast microscopy (B) and was stained for K15 (C), showing confluent staining in the basal epidermis as well as the bulge region. (Dashed lines $=$ basement membrane, epi $=$ epidermis).

suggest that the number and location of stem cells within skin may be a contributing factor in rapid fetal scarless wound healing and the decreased healing in older adults. Future studies that correlate wound healing with age and epithelial stem cells may shed more light on this issue.

\section{CONCLUSION}

It is clear that adult cutaneous stem cells will become a useful resource for therapeutic applications due to their plasticity and accessibility. Although we and others have characterized the cellular and molecular mechanisms that define a cutaneous stem cell and control their fate, relatively little is known about the differences between fetal and adult tissuespecific stem cells and the interactions between stem cells and their environment which also change with aging. Determining the function of genes in controlling stem cell behavior should advance our understanding of the normal physiology of stem cells. In addition, a better understanding of cell signaling during wound-induced activation of fetal and adult stem cells should lead to improved therapeutic strategies to make adult skin heal more like the fetal tissue.

\section{REFERENCES}

1. Montagna W 1962 The Structure and Function of Skin. Academic Press, New York

2. Taylor G, Lehrer MS, Jensen PJ, Sun TT, Lavker RM 2000 Involvement of follicular stem cells in forming not only the follicle but also the epidermis. Cell 102:451-461

3. Ito M, Liu Y, Yang Z, Nguyen J, Liang F, Morris RJ, Cotsarelis G 2005 Stem cells in the hair follicle bulge contribute to wound repair but not to homeostasis of the epidermis. Nat Med 11:1351-1354

4. Bullard KM, Longaker MT, Lorenz P 2003 Fetal wound healing: current biology. World J Surg 27:54-61

5. Zouboulis CC 2004 Acne and sebaceous gland function. Clin Dermatol 22:360-366

6. Cotsarelis G 1997 The hair follicle: dying for attention. Am J Pathol 151:1505-1509

7. Cotsarelis G, Sun TT, Lavker RM 1990 Label-retaining cells reside in the bulge area of pilosebaceous unit: implications for follicular stem cells, hair cycle, and skin carcinogenesis. Cell 61:1329-1337

8. Lyle S, Christofidou-Solomidou M, Liu Y, Elder DE, Albelda S, Cotsarelis G 1998 The $\mathrm{C} 8 / 144 \mathrm{~B}$ monoclonal antibody recognizes cytokeratin 15 and defines the location of human hair follicle stem cells. J Cell Sci 111:3179-3188

9. Morris RJ, Liu Y, Marles L, Yang Z, Trempus C, Li S, Lin JS, Sawicki JA, Cotsarelis G 2004 Capturing and profiling adult hair follicle stem cells. Nat Biotechnol 22:411-417

10. Nishimura EK, Granter SR, Fisher DE 2005 Mechanisms of hair graying: incomplete melanocyte stem cell maintenance in the niche. Science 307:720-724

11. Bickenbach JR 1981 Identification and behavior of label-retaining cells in oral mucosa and skin. J Dent Res 60:1611-1620

12. Morris RJ, Fischer SM, Slaga TJ 1985 Evidence that the centrally and peripherally located cells in the murine epidermal proliferative unit are two distinct cell populations. J Invest Dermatol 84:277-281

13. Morris RJ, Potten CS 1999 Highly persistent label-retaining cells in the hair follicles of mice and their fate following induction of anagen. J Invest Dermatol 112:470-475

14. Lyle S, Christofidou-Solomidou M, Liu Y, Elder DE, Albelda S, Cotsarelis G 1999 Human hair follicle bulge cells are biochemically distinct and possess an epithelial stem cell phenotype. J Investig Dermatol Symp Proc 4:296-301

15. Barrandon Y, Green H 1987 Three clonal types of keratinocyte with different capacities for multiplication. Proc Natl Acad Sci USA 84:2302-2306

16. Jones PH, Watt FM 1993 Separation of human epidermal stem cells from transit amplifying cells on the basis of differences in integrin function and expression. Cell 73:713-724

17. Kobayashi K, Rochat A, Barrandon Y 1993 Segregation of keratinocyte colonyforming cells in the bulge of the rat vibrissa. Proc Natl Acad Sci USA 90:7391-7395

18. Jones PH, Harper S, Watt FM 1995 Stem cell patterning and fate in human epidermis. Cell 80:83-93

19. Rochat A, Kobayashi K, Barrandon Y 1994 Location of stem cells of human hair follicles by clonal analysis. Cell 76:1063-1073

20. Moll I 1995 Proliferative potential of different keratinocytes of plucked human hair follicles. J Invest Dermatol 105:14-21

21. Roh C, Tao Q, Photopoulos C, Lyle S 2005 In vitro differences between keratinocyte stem cells and transit-amplifying cells of the human hair follicle. J Invest Dermatol 125:1099-1105

22. Oshima H, Rochat A, Kedzia C, Kobayashi K, Barrandon Y 2001 Morphogenesis and renewal of hair follicles from adult multipotent stem cells. Cell 104:233-45

23. Roh C, Tao Q, Lyle S 2004 Dermal papilla-induced hair differentiation of adult epithelial stem cells from human skin. Physiol Genomics 19:207-217

24. Li A, Simmons PJ, Kaur P 1998 Identification and isolation of candidate human keratinocyte stem cells based on cell surface phenotype. Proc Natl Acad Sci USA 95:3902-3907

25. Tani H, Morris RJ, Kaur P 2000 Enrichment for murine keratinocyte stem cells based on cell surface phenotype. Proc Natl Acad Sci USA 97:10960-10965

26. Ivanova NB, Dimos JT, Schaniel C, Hackney JA, Moore KA, Lemischka IR 2002 A stem cell molecular signature. Science 298:601-604

27. Ramalho-Santos M, Yoon S, Matsuzaki Y, Mulligan RC, Melton DA 2002 "Stemness": transcriptional profiling of embryonic and adult stem cells. Science 298:597-600

28. Liu Y, Lyle S, Yang Z, Cotsarelis G 2003 Keratin 15 promoter targets putative epithelial stem cells in the hair follicle bulge. J Invest Dermatol 121:963-968

29. Pellegrini G, Dellambra E, Golisano O, Martinelli E, Fantozzi I, Bondanza S, Ponzin D, McKeon F, De Luca M 2001 p63 identifies keratinocyte stem cells. Proc Natl Acad Sci USA 98:3156-3161

30. Zhu AJ, Watt FM 1999 Beta-catenin signalling modulates proliferative potential of human epidermal keratinocytes independently of intercellular adhesion. Development 126:2285-2298

31. Ghali L, Wong ST, Tidman N, Quinn A, Philpott MP, Leigh IM 2004 Epidermal and hair follicle progenitor cells express melanoma-associated chondroitin sulfate proteoglycan core protein. J Invest Dermatol 122:433-442

32. Tumbar T, Guasch G, Greco V, Blanpain C, Lowry WE, Rendl M, Fuchs E 2004 Defining the epithelial stem cell niche in skin. Science 303:359-363

33. Xu X, Lyle S, Liu Y, Solky B, Cotsarelis G 2003 Differential expression of cyclin D1 in the human hair follicle. Am J Pathol 163 (3):969-978

34. Magnaldo T, Barrandon Y 1996 CD24 (heat stable antigen, nectadrin), a novel keratinocyte differentiation marker, is preferentially expressed in areas of the hair follicle containing the colony-forming cells. J Cell Sci. 109:3035-3045

35. Gosain A, DiPietro LA 2004 Aging and wound healing. World J Surg 28:321-326

36. Rowlatt U 1979 Intrauterine wound healing in a 20-week human fetus. Virshows Arch A Pathol Anat Histol 381:353-361

37. Tomic-Canic M, Komine M, Freedberg IM, Blumenberg M 1998 Epidermal signal transduction and transcription factor activation in activated keratinocytes. J Dermtol Sci 17:167-181 